\title{
Distribution Center Consolidation Games
}

\author{
Flip Klijn* $\quad$ Marco Slikker ${ }^{\dagger}$
}

February 6, 2004

\begin{abstract}
We study the location-inventory model as introduced by Teo et al. (2001) to analyze the impact of consolidation of distribution centers on facility and inventory costs. We extend their result on profitability of consolidation. We associate a cooperative game with each location-inventory situation and prove that this game has a nonempty core for identical and independent demand processes. This illustrates that consolidation does not only lower joint costs (which was shown by Teo et al. (2001)), but it allows for a stable division of the minimal costs as well.
\end{abstract}

\section{Introduction}

During the last decade, consolidation of distribution centers (DCs) has turned out to be an excellent strategy in global logistics management. One of the explanations that is often cited to rationalize its rate of success is the reduction of facility and inventory costs that comes with the consolidation and centralization of distribution operations.

Facility location problems as well as inventory control have each been studied extensively, but separately, in a large literature. In fact, in several studies on facility location problems the demands processes are often (quite unrealistically) oversimplified for the sake of tractability. On the other hand, in the literature on inventory control there has not been much integration of other supply chain activities until recently. We refer the reader to Teo et al. (2001) for an overview on papers in which both lines of research do meet.

As a matter of fact, in the paper mentioned above both aspects are dealt with simultaneously. The paper can thus be viewed as a study of a facility location model that

${ }^{*}$ Corresponding author. Institut d'Anàlisi Econòmica (CSIC), Campus UAB, 08193 Bellaterra (Barcelona), Spain; e-mail: fklijn@pareto.uab.es. F. Klijn's research is supported by a Ramón y Cajal contract of the Spanish Ministerio de Ciencia y Tecnología. His work is also partially supported by Research Grant BEC2002-02130 from the Spanish Ministerio de Ciencia y Tecnología and by the Barcelona Economics Program of CREA.

${ }^{\dagger}$ Department of Technology Management, Technische Universiteit Eindhoven, P.O. Box 513, 5600 MB Eindhoven, The Netherlands; e-mail: M.Slikker@tm.tue.nl. 
captures the impact of the inventory related costs with more sophistication. In fact, Teo et al. (2001) allow for stochastic demands at the customer locations, which brings along the analytical problem of not having at hand closed form expressions for the optimal inventory costs for most demand distributions. As they pointed out, their analysis was facilitated in a great deal by recent advances in the area of stochastic inventory theory, mainly due to the papers by Zheng (1992) and Gallego (1998).

In the location-inventory problem that Teo et al. (2001) study there is a firm with a production plant producing a single product for a collection of aggregated demand points (customers). There are a number of possible locations to set up regional distribution centers, from which the customers are to be served. The main problem the firm faces is to choose the locations so as to minimize total facility and inventory costs. Some assumptions (indifference of the customers with respect to the DCs and constant outbound transportation costs) are made to isolate the effect of ordering costs and replenishment leadtimes on the consolidation strategy. One of their main results is that consolidation leads to lower facility investment and inventory costs if the demands are identically and independently distributed.

In this paper, we study the location-inventory problem described above and build on several results provided by Zheng (1992) and Teo et al. (2001) to extend the profitability result in the latter work. Our main result says that the minimal total costs (which correspond to the consolidation into a central DC) can also be divided in a stable way among the DC locations. There are (at least) two possible interpretations of the existence of a stable allocation of the minimal total costs. In the first place, it means that there is a way to divide the costs so that no set of DC locations can protest against the consolidation strategy arguing that they could take care of their own customers at less costs. Another interpretation is possible if we assume that the DCs have already been set up. In this case the set up costs, which are amortized over time, can be reinterpreted as being the costs involved in running a DC. The existence of a stable cost allocation then implies that costs can be divided in such a way that no set of DC locations can object against the firm centralizing its DCs into one central DC.

The allocation of costs is one of the main issues that are addressed in cooperative game theory. Cooperative game theory deals with situations involving several agents who can benefit from cooperation. Establishing a relation between location-inventory problems and cooperative games then enables us to apply cooperative game theory to study the existence of core allocations, i.e., stable cost allocations. Not surprisingly, cooperative game theory has been invoked before for the same reason in several other studies on inventory problems. Cooperative games associated with news-vendor situations were studied in Hartman et al. (2000), Slikker et al. (2001), and Müller et al. (2002). The last two of these papers independently established the existence of stable allocations in news-vendor situations. Moreover, they provided conditions under which news-vendor games are convex. Thus they neatly complement the results of Gerchak and Gupta (1991), Robinson (1993), and Hartman and Dror (1996) on allocation rules in continuous review single period inventory systems with complete back-ordering. Meca et al. (1999) studied cooperative games associated with inventory situations with deterministic demand processes. They focus 
on the information that is revealed by the companies and proportional cost allocation mechanisms. Finally, Borm et al. (2001) recently provided a survey of cooperative games associated with operations research situations. They focus on five different natures of the underlying optimization problem, one of which being 'inventory'.

The rest of the paper is organized as follows. We start in Section 2 with some preliminaries on the stochastic $(Q, r)$-model and cooperative game theory. Then, in Section 3, we recall the location-inventory model of Teo et al. (2001), and introduce the class of associated DC consolidation games. In Section 4 we present our result on the balancedness of DC consolidation games in case the demands are identically and independently distributed.

\section{Preliminaries}

In this section we recall some concepts and definitions of $(Q, r)$-policies and cooperative game theory.

The location-inventory model that we consider includes an order quantity/reorder point system, i.e., a $(Q, r)$-system. More precisely, we study a single-item continuousreview inventory system, where stochastic demands arrive at mean rate $\mu$ per unit time. Replenishment orders are delivered after a positive fixed leadtime $L$. All stockouts are backordered. The inventory costs consist of an ordering cost $k(Q)$ per order, $Q$ being the ordering size, and proportional inventory holding (penalty) costs accumulating at a constant rate $h(p)$ per unit stock (backorder) per unit time. The ordering cost $k(Q)$ is modeled as a piece-wise linear concave increasing function of the ordering size $Q$ to account for the economies of scale in purchasing and transportation. We concentrate on minimizing the long run average total costs per unit time.

Under mild conditions on the stochastic demand (see Serfozo and Stidham (1982) and Browne and Zipkin (1991) for detailed discussions) the long run average total costs per unit time takes the following form:

$$
C(Q, r)=C(Q, r, \mu)=\frac{\mu k(Q)+\int_{r}^{r+Q} G(y) d y}{Q} \text { for all } Q, r>0,
$$

where $G(y)$ is the rate at which the expected inventory costs accumulate at time $t+L$ when the inventory position at time $t$ equals $y \in \mathbb{R}$, i.e., ${ }^{1}$

$$
G(y)=E\left(h(y-D)^{+}+p(D-y)^{+}\right)
$$

$D$ being the sum of demands that occur during the time interval $(t, t+L]$. The term $\frac{\lambda k(Q)}{Q}$ in (1) reflects the average ordering cost. The other term, $\frac{\int_{r}^{r+Q} G(y, \mu) d y}{Q}$, reflects the average holding and stockout cost of the system, as the inventory level can be shown to be uniformly distributed between $r$ and $r+Q$ (cf. Zheng (1992)).

\footnotetext{
${ }^{1}$ For $x \in \mathbb{R}$ we write $x^{+}=\max \{0, x\}$.
} 
The following (technical) results are due to Zheng (1992) and facilitate much of our analysis later on. Define

$$
r(Q):=\operatorname{argmin}_{r} \int_{r}^{r+Q} G(y) d y \text { for all } Q>0 .
$$

Then, $r(Q)$ is the optimal reorder point when the order quantity is fixed at $Q$. Zheng (1992) showed that

$$
G(r(Q))=G(r(Q)+Q) \text { for all } Q>0 .
$$

Moreover, he proved that the functions $H$ and $S$ defined by

$$
H(Q):= \begin{cases}\min _{y} G(y) & \text { if } Q=0 \\ G(r(Q)) & \text { if } Q>0\end{cases}
$$

and

$$
S(Q):= \begin{cases}0 & \text { if } Q=0 ; \\ \int_{r(Q)}^{r(Q)+Q} G(y) d y=\int_{0}^{Q} H(y) d y & \text { if } Q>0,\end{cases}
$$

are both convex in $Q$. Hence, using $S(0)=0$, we have that

$$
S(\alpha Q) \leq \alpha S(Q) \text { for all } Q>0 \text { and } 0 \leq \alpha \leq 1 .
$$

The other ingredient of this paper is cooperative game theory of which we recall the necessary basic definitions below. A cooperative (cost) game with transferable utility (game, for short) is a pair $(N, c)$, where $N=\{1, \ldots, n\}$ is the player set and $c$ the characteristic function, which assigns to every subset $S \subseteq N$ a value $c(S)$, with $c(\emptyset)=0$. The number $c(S)$ represents the minimal costs for coalition $S$ when all its members decide to cooperate. The subgames of $(N, c)$ are the games $\left(S, c_{\mid S}\right)$ where $S \subseteq N$ and $c_{\mid S}: S \rightarrow \mathbb{R}$ is the restriction of the map $c$ to the coalition $S$.

Cooperative game theory focuses on fair division rules for the value of $c(N)$ of the grand coalition. A core allocation $x=\left(x_{i}\right)_{i \in N} \in \mathbb{R}^{N}$ divides the value $c(N)$ among the players in such a way that no coalition has an incentive to split off, i.e.,

$$
x(N)=c(N) \quad \text { and } \quad x(S) \leq c(S) \quad \text { for all } S \subseteq N,
$$

where $x(S)=\sum_{i \in S} x_{i}$ for all $S \subseteq N$. The core $\operatorname{Core}(N, c)$ is the (possibly empty) set of core allocations.

A map $\lambda: 2^{N} \backslash\{\emptyset\} \rightarrow[0,1]$ is called balanced if for all $i \in N$ it holds that $\sum_{S \subseteq N: i \in S} \lambda(S)=$ 1. A cost game $(N, c)$ is called balanced if for each balanced map $\lambda$ we have

$$
\sum_{S \subseteq N} \lambda(S) c(S) \geq c(N)
$$


Bondareva (1963) and Shapley (1967) proved $^{2}$ independently that non-emptiness of the core and balancedness of a game are equivalent, i.e., for a game $(N, c)$ we have

$$
\operatorname{Core}(N, c) \neq \emptyset \Leftrightarrow(N, c) \text { is balanced. }
$$

A game is called totally balanced if all its subgames are balanced.

\section{The model}

In this section we recall the location-inventory problem as defined by Teo et al. (2001). Moreover, we introduce the class of consolidation games which are cost games associated with the location-inventory problems.

In the location-inventory problem, there is a set $M=\{1,2, \ldots, m\}$ of demand points. We assume that the demand for the product at demand point $j$ can be modeled by a continuous stochastic process $\left\{d_{j}(t): t \geq 0\right\}$, with mean rate $\mu_{j}$ per unit time.

The demands are to be served by $n$ possible distribution centers, which are indexed by $1, \ldots, n$. Let $N=\{1, \ldots, n\}$ be the set of possible DC locations. We assume that the demand points (i.e., customers) are indifferent about where their orders are shipped from, and also that the outbound transportation costs do not depend on where the orders are shipped from. We assume that initially each DC $i$ is to serve aggregate demand $\sum_{j \in M} x_{i j}^{0} d_{j}(t)$, where the $\left(x_{i j}^{0}\right)_{i \in N, j \in M}$ satisfy $x_{i j}^{0} \in\{0,1\}(i \in N, j \in M)$ and $\sum_{i=1}^{n} x_{i j}^{0}=1$ $(j \in M)$. So, the demands at each demand point are exclusively served from a single DC.

A group (coalition) $S \subseteq N$ of distribution centers may decide to cooperate and reassign the initial demands within the coalition. A demand re-assignment for $S$ is a matrix $X^{S}=\left(x_{i j}^{S}\right)_{i \in S, j \in M}$ that satisfies $x_{i j}^{S} \in\{0,1\} \quad(i \in S, j \in M)$ and $\sum_{i \in S} \sum_{j \in M} x_{i j}^{S}=$ $\sum_{i \in S} \sum_{j \in M} x_{i j}^{0}$. Let $\Pi^{S}$ be the set of demand re-assignments for $S$. If for a given demand re-assignment $X^{S}$ it holds that $X_{i}^{S}:=\sum_{j=1}^{m} x_{i j}>0$, then DC $i$ is set up for which it incurs a fixed setup cost $f_{i}$. We assume that $f_{i}$ is a per unit time charge, which then also could be treated as and/or include the direct variable costs in running a DC. Let $L_{i}$ be the leadtime to supply from the plant to DC $i$. The inventory costs at DC $i$ include an ordering cost $k_{i}(Q)$ per order, $Q$ being the ordering size, and proportional inventory holding (penalty) costs accumulating at a constant rate $h_{i}\left(p_{i}\right)$ per unit stock (backorder) per unit time. The ordering cost $k_{i}(Q)$ is modeled as a piece-wise linear concave increasing function of the ordering size $Q$ to account for the economies of scale in purchasing and transportation.

If a coalition $S \subseteq N$ of distribution centers decides to cooperate, i.e., re-assign the initial demand within $S$ to minimize costs, then it follows readily that the optimal long

\footnotetext{
${ }^{2}$ In fact, Bondareva (1963) and Shapley (1967) proved that a cost savings game $(N, v)$ is balanced (i.e., there is an allocation $x=\left(x_{i}\right)_{i \in N} \in \mathbb{R}^{N}$ such that $x(S) \geq v(S)$ for all $S \subseteq N$ with equality for $S=N)$ if and only if for each balanced map $\lambda$ we have $\sum_{S \subset N} \lambda(S) v(S) \leq v(N)$. This is easily shown to be equivalent with the above statement.
} 
run average costs for $S$ equal

$$
c(S):=\min _{X^{S} \in \Pi^{S}}\left\{\sum_{i \in S}\left[f_{i} \chi\left(x_{i}^{S}>0\right)+\min _{r, Q} C\left(r, Q, \sum_{j \in M} x_{i j}^{S} \mu_{j}\right)\right]\right\},
$$

where $\chi$ is the indicator function and $C$ the cost function as defined in (1).

\section{Balancedness}

In this section we restrict ourselves to identically and independently distributed (i.i.d.) demand processes. We prove that the associated consolidation games are balanced, i.e., allow for stable divisions of the joint costs.

Since demands are i.i.d. we can assume without loss of generality that $\mu_{j}=1$ for all demand points $j \in M$. Let $n_{i}:=x_{i}^{0}=\sum_{j \in M} x_{i j}^{0}$ be the aggregate demand that originally would be served by DC $i \in N$. Define $n_{S}:=\sum_{i \in S} n_{i}$ for all $S \subseteq N$. Recall that $x_{i j}^{0} \in\{0,1\}$ for all $i \in N, j \in M$, so that $n_{S} \in \mathbb{N}$ for all $S \subseteq N$. Note that $n_{N}=m$.

Let us now consider the $(Q, r)$-system that corresponds to a distribution center that serves a set $M^{\prime} \subseteq M$ of demand points. Since the demand points are i.i.d. we can define $G\left(y, m^{\prime}\right)$ as the rate at which the expected inventory costs accumulate at time $t+L$ when the inventory position at time $t$. The next result is due to Teo et al. (2001) (page 102).

Lemma 4.1 (Teo et al. (2001)) For all $y \in \mathbb{R}$ and $0 \leq m^{\prime} \leq m$ we have $\frac{m^{\prime}}{m} G(y, m) \leq$ $G\left(\frac{m^{\prime}}{m} y, m^{\prime}\right)$.

Theorem 4.2 Consolidation games have a non-empty core when the demand processes are i.i.d..

Proof. Let $\lambda: 2^{N} \backslash\{\emptyset\} \rightarrow[0,1]$ be a balanced map.

Let $S \subseteq N$. From Theorem 1 in Teo et al. (2001) we know that an optimal strategy for coalition $\mathrm{S}$ is consolidation at some $\mathrm{DC}$ in $S$, say $i_{S}$.

Consider the strategy for the grand coalition in which with probability $\lambda(S) \frac{n_{S}}{n_{N}}(S \subseteq$ $N)$ consolidation at $i_{S}$ takes place. Equivalently, a DC $i \in N$ is picked with probability $\sum_{S \subseteq N: i=i_{S}} \lambda(S) \frac{n_{S}}{n_{N}}$. Note that $\sum_{S \subseteq N} \lambda(S) \frac{n_{S}}{n_{N}}=\frac{1}{n_{N}} \sum_{S \subseteq N} \lambda(S) n_{S}=\frac{1}{n_{N}} \sum_{S \subseteq N} \sum_{i \in S} \lambda(S) n_{i}=$ $\frac{1}{n_{N}} \sum_{i \in N} \sum_{S: i \in S} \lambda(S) n_{i}=\frac{1}{n_{N}} \sum_{i \in N} n_{i} \sum_{S: i \in S} \lambda(S)=\frac{1}{n_{N}} \sum_{i \in N} n_{i}=1$.

Since this strategy is a weighted average of (pure) consolidation strategies, the asso- 
ciated costs majorize the optimal costs of the grand coalition. Hence,

$$
\begin{aligned}
c(N) & \leq \sum_{S \subseteq N} \lambda(S) \frac{n_{S}}{n_{N}}\left[f_{i_{S}}+\min _{r, Q} \frac{k_{i_{S}}(Q) n_{N}+\int_{r}^{r+Q} G_{i_{S}}\left(y, n_{N}\right) d y}{Q}\right] \\
& =\sum_{S \subseteq N} \lambda(S)\left[\frac{n_{S}}{n_{N}} f_{i_{S}}+\min _{r, Q} \frac{n_{S}}{n_{N}} \frac{k_{i_{S}}(Q) n_{N}+\int_{r}^{r+Q} G_{i_{S}}\left(y, n_{N}\right) d y}{Q}\right] \\
& \leq \sum_{S \subseteq N} \lambda(S)\left[f_{i_{S}}+\min _{r, Q} \frac{n_{S}}{n_{N}} \frac{k_{i_{S}}(Q) n_{N}+\int_{r}^{r+Q} G_{i_{S}}\left(y, n_{N}\right) d y}{Q}\right] \\
& =\sum_{S \subseteq N} \lambda(S)\left[f_{i_{S}}+\min _{r, Q} \frac{k_{i_{S}}(Q) n_{S}+\int_{r}^{r+Q} \frac{n_{S}}{n_{N}} G_{i_{S}}\left(y, n_{N}\right) d y}{Q}\right] \\
& \leq \sum_{S \subseteq N} \lambda(S)\left[f_{i_{S}}+\min _{r, Q} \frac{k_{i_{S}}(Q) n_{S}+\int_{r}^{r+Q} G_{i_{S}}\left(\frac{n_{S}}{n_{N}} y, n_{S}\right) d y}{Q}\right] \\
& =\sum_{S \subseteq N} \lambda(S)\left[f_{i_{S}}+\min _{r, Q} \frac{k_{i_{S}}(Q) n_{S}+\frac{n_{N}}{n_{S}} \int_{n_{S} r / n_{N}}^{n_{S}(r+Q) / n_{N}} G_{i_{S}}\left(\frac{n_{S}}{n_{N}} y, n_{S}\right) d y}{Q}\right] \\
& =\sum_{S \subseteq N} \lambda(S)\left[f_{i_{S}}+\min _{r, Q} \frac{k_{i_{S}}(Q) n_{S}+\frac{n_{N}}{n_{S}} \int_{r}^{r+n_{S} Q / n_{N}} G_{i_{S}}\left(\frac{n_{S}}{n_{N}} y, n_{S}\right) d y}{Q}\right] \\
& \leq \sum_{S \subseteq N} \lambda(S)\left[f_{i_{S}}+\min _{r, Q} \frac{k_{i_{S}}(Q) n_{S}+\int_{r}^{r+Q} G_{i_{S}}\left(y, n_{S}\right) d y}{Q} \lambda(S) c(S) .\right. \\
& \left.=\sum_{S \subseteq N}\right] \\
& {[}
\end{aligned}
$$

The third inequality follows from Lemma 4.1 with $m^{\prime}=n_{S}$. The last inequality follows from (2) with $\alpha=\frac{n_{S}}{n_{N}}$. The last equality follows from the optimality for $S$ to consolidate into DC $i_{S}$.

We conclude that the consolidation game is balanced and hence, using the result by Bondareva (1963) and Shapley (1967), that it has a non-empty core.

There are (at least) two possible interpretations of Theorem 4.2. In the first place, it means that there is a way to divide the minimal total costs so that no set of DC locations can protest against the consolidation strategy arguing that they could take care of their own customers at less costs. Another interpretation is possible if we assume that the DCs have already been set up. In this case the set up costs, which are amortized over time, can be reinterpreted as being the costs involved in running a DC. The existence of a stable cost allocation then implies that costs can be divided in such a way that no set of DC locations can object against the firm centralizing its DCs into one central DC. 
The following result follows immediately by noting that a subgame $\left(S, c_{\mid S}\right)$ of a consolidation game $(N, c)$ is again a consolidation game, associated with the original locationinventory problem restricted to $S$.

Corollary 4.3 Every consolidation game is totally balanced when the demand processes are i.i.d..

\section{References}

Bondareva, O.N. (1963). "Some Applications of the Methods of Linear Programming to the Theory of Cooperative Games," (in Russian) Problemy Kibernetiki, 10, 119-139.

Borm, P., Hamers, H., and Hendrickx, R. (2001). "Operations Research Games: A Survey," TOP, 9, 139-198.

Browne, S. and Zipkin, P. (1991). "Inventory Models with Continuous, Stochastic Demands," Annals of Applied Probability, 1, 419-435.

Gallego G. (1998). "New Bounds and Heuristics for $(Q, r)$ Policies," Management Science, 44, 219-233.

Gerchak, Y. and Gupta, D. (1991). "Technical Note: On Apportioning Costs to Customers in Centralized Continuous Review Inventory Systems," Journal of Operations Management, 10, 546-551.

Hartman, B.C. and Dror, M. (1996). "Cost Allocation in Continuous Review Inventory Models," Naval Research Logistics Quarterly, 43, 549-561.

Hartman, B.C., Dror, M., and Shaked, M. (2000). "Cores of Inventory Centralization Games," Games and Economic Behavior, 31, 26-49.

Meca, A., Timmer, J., García-Jurado, I., and Borm, P. (1999). "Inventory Games," CentER Discussion Paper 9953, Tilburg University, Tilburg, The Netherlands. To appear in European Journal of Operational Research.

Müller, A., Scarsini M., and Shaked M. (2002). "The Newsvendor Game has a nonempty Core," Games and Economic Behavior, 38, 118-126.

Robinson, L. (1993). "A Comment on Gerchak and Gupta's: On Apportioning Costs to Customers in Centralized Continuous Review Inventory Systems," Journal of Operations Management, 11, 99-102.

Serfozo, R. and Stidham, S. (1982). "Semi-Stationary Clearing Processes," Stochastic 
Process and Applications, 6, 165-178.

Shapley, L.S. (1967). "On Balanced Sets and Cores," Naval Research Logistics Quarterly, 14, 453-460.

Slikker, M., Fransoo, J., and Wouters, M. (2001). "Joint Ordering in Multiple NewsVendor Problems: A Game-Theoretical Approach," Beta Working Paper WP-64, Department of Technology Management, Eindhoven University of Technology, Eindhoven, The Netherlands.

Teo, C.P., Ou J., and Goh, M. (2001). "Impact on Inventory Costs with Consolidation of Distribution Centers," IIE Transactions, 33, 99-110.

Zheng Y.S. (1992). "On Properties of Stochastic Inventory Systems," Management Science, 38, 87-103. 